\section{Fatores sociodemográficos e clínicos associados ao tempo para o início do tratamento de câncer de cólon e reto no Brasil, 2006-2015}

\author{
Sociodemographic and clinical factors associated \\ with time to treatment for colorectal cancer in \\ Brazil, 2006-2015
}

\author{
Factores sociodemográficos y clínicos \\ relacionados con el tiempo para el inicio \\ del tratamiento de cáncer de colon y \\ recto en Brasil, 2006-2015
}

Mariana Araujo Neves Lima 1

Daniel Antunes Maciel Villela 2

\section{Resumo}

O câncer de cólon e reto apresenta alta incidência mundialmente, porém a letalidade da doença é maior em países em desenvolvimento. O objetivo deste estudo é analisar fatores sociodemográficos e clínicos associados ao atraso para o início de tratamento de câncer de cólon e reto em hospitais no Brasil. Trata-se de estudo retrospectivo com dados dos registros hospitalares de câncer no Brasil de 2006 a 2015. O desfecho analisado é o tempo para início do tratamento de câncer de cólon e reto e possíveis associações entre variáveis sociodemográficas e referentes a fatores clínicos. Observaram-se disparidades no tempo para início do tratamento de acordo com estratos sociodemográficos e regiões geográficas. Há maior chance de atraso para o início do tratamento de câncer de cólon em pacientes com idade acima de 50 anos, de raça/cor de pele preta $(O R=1,50$; IC95\%: 1,21-1,84) e parda $(O R=1,28$; IC95\%: 1,17-1,42), analfabetos $(O R=1.50$; IC95\%: 1,19-1,90) ou com baixa escolaridade e cujo tratamento ocorreu em um município distinto de sua residência $(O R=1,25$; IC95\%: 1,14-1,38). Em pacientes com câncer de reto, há maior chance de atraso para o início do tratamento entre os casos com idade acima de 50 anos, de raça/cor de pele preta $(O R=1,44 ;$ IC95\%: 1,20-1,72) e parda $(O R=1,29$; IC95\%: 1,19-1,39), analfabetos (OR = 1,71; IC95\%: 1,40-2,09) ou com baixa escolaridade e cujo tratamento ocorreu em um município distinto de sua residência $(O R=1,35$; IC95\%: 1,25-1,47). Como conclusão, maior atenção deve ser destinada a reduzir o tempo para iniciar o tratamento nas regiões desfavorecidas e nos estratos identificados com barreiras de acesso ao tratamento em tempo oportuno.

Neoplasias do Cólon; Neoplasias Retais; Tempo para o Tratamento; Registros Hospitalares; Acesso aos Serviços de Saúde

\author{
Correspondência \\ D. A. M. Villela \\ Programa de Computação Científica, Fundação Oswaldo Cruz. \\ Av. Brasil 4365, Rio de Janeiro, RJ 21040-360, Brasil. \\ daniel.villela@fiocruz.br \\ 1 Escola Nacional de Saúde Pública Sergio Arouca, Fundação \\ Oswaldo Cruz, Rio de Janeiro, Brasil. \\ 2 Programa de Computação Científica, Fundação Oswaldo \\ Cruz, Rio de Janeiro, Brasil.
}




\section{Introdução}

O câncer de cólon e reto é o terceiro tipo de câncer mais incidente no mundo, sendo três vezes mais frequente em países desenvolvidos. Mundialmente, 551,3 mil óbitos foram devidos ao câncer de cólon e 310,4 mil decorrentes do câncer de reto em 2018 1. No Brasil, são esperados 19,63 casos/100 mil homens e 19,03 casos/100 mil mulheres em 2020 a 2022 2. Com o aumento do número de casos de câncer, houve uma crescente demanda por melhoria no diagnóstico e tratamento. No entanto, o tempo de espera para início de tratamento ainda é considerado longo no Brasil, portanto sua redução permanece como problema recorrente 3,4,5.

Estima-se para o câncer de cólon e reto, nas regiões Sudeste e Sul, uma alta incidência no triênio 2020-2022. Para homens, a estimativa corresponde a 28,6 casos/100 mil no Sudeste e 25,1 casos/100 mil na Região Sul. Para mulheres, a estimativa é de 26,1 casos/100 mil na Região Sudeste e 23,65 casos/100 mil na Região Sul 2. Nessas regiões, há maior prevalência de fatores de risco para a doença, tais como idade (50 anos ou mais), obesidade, sedentarismo, dieta inadequada, tabagismo e consumo elevado de álcool 6,7.

A taxa de mortalidade por câncer colorretal nos países desenvolvidos encontra-se em declínio, apesar de sua incidência ainda ser alta em alguns casos. Tal declínio tem sido frequentemente associado à presença de programas de rastreamento consolidados e adoção de melhorias no tratamento 8 . No Brasil, a mortalidade por câncer colorretal apresenta aumento. Em 2006, a taxa de mortalidade ajustada por idade era de 6,65 óbitos por 100 mil habitantes, ao passo que em 2015 ocorreram 16.291 óbitos, totalizando uma taxa de 7,48 óbitos por 100 mil habitantes 9.

Em 2012, foi sancionada a Lei Federal no 12.732 no Brasil, a qual visa garantir que todo paciente com neoplasia maligna tem direito de iniciar o tratamento no Sistema Único de Saúde (SUS) em até 60 dias 10. Além do desafio de garantir acesso ao SUS em tempo oportuno, no Brasil há evidências de desigualdade no tempo de espera em estudos relacionados ao tratamento do câncer de mama ${ }^{3}$, de colo do útero 4 e de próstata 5 . Tais estudos apontam heterogeneidades significativas no tempo de espera quanto a idade, raça/cor da pele, estado conjugal, escolaridade, região de residência, tipo de tratamento, estadiamento e distância entre residência e hospital 3,4,5.

No Brasil, dadas essas heterogeneidades, é importante avaliar fatores relacionados ao atraso no tempo de espera para início de tratamento do câncer colorretal. Apesar de sua alta incidência, esses fatores ainda carecem de estudo detalhado em nível nacional, de macrorregiões e de estados. Esta pesquisa tem como objetivo analisar fatores associados ao tempo para início do tratamento de câncer de cólon e reto em hospitais no Brasil e nas regiões brasileiras durante os anos de 2006 a 2015.

\section{Métodos}

\section{População de estudo}

Para compor a coorte retrospectiva do estudo, foram selecionados pacientes diagnosticados com neoplasia do cólon (CID-10: C18) e reto (CID-10: C19 e C20) e classificados como casos analíticos. Segundo o manual de registro hospitalar de câncer 11, esses casos correspondem a pacientes cujo tratamento principal foi estabelecido e realizado pela instituição que o registrou, evitando, assim, duplicidade. Por sua vez, casos não analíticos se referem a pacientes que chegaram ao hospital para complementação do tratamento ou sem possibilidade terapêutica.

Foram selecionados os pacientes com início de tratamento entre os anos de 2006 a 2015, considerando a implantação da Portaria no 741, em dezembro de 2005, que instituiu a Política Nacional de Atenção Oncológica 12. A fim de minimizar erros de digitação nas datas de diagnóstico e tratamento, foram excluídos os casos que foram registrados com tratamento anterior e aqueles com valor negativo para o intervalo de tempo entre diagnóstico e tratamento (Figura 1). 
Figura 1

Fluxograma da população de estudo de acordo com os critérios de inclusão e exclusão.

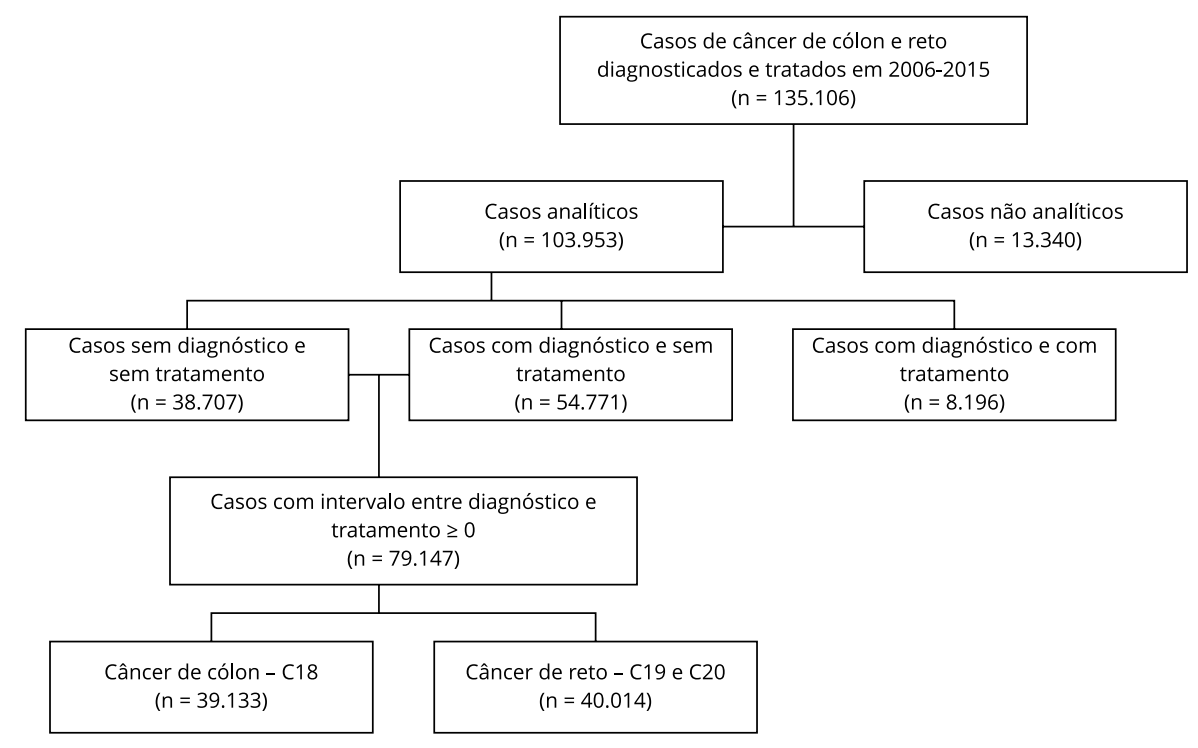

\section{Fonte de dados}

Os dados analisados são oriundos de unidades com Registro Hospitalar de Câncer. Para elaboração do banco de dados, foram acessadas duas fontes de informação: o sítio do Integrador do Registro Hospitalar de Câncer, gerido pelo Instituto Nacional de Câncer José Alencar Gomes da Silva (INCA. https:// www.inca.gov.br/), e o sítio da Fundação Oncocentro de São Paulo (FOSP. http://www.fosp.saude.sp. gov.br/) para obter dados referentes às unidades do Estado de São Paulo. Anualmente ocorre o envio de tais dados ao INCA, o qual realiza a consolidação desses números e disponibiliza a base de dados sem a identificação dos pacientes 11 . O acesso aos sítios eletrônicos ocorreu em 25 de junho de 2018.

\section{Variáveis de estudo}

O desfecho do estudo consistiu no intervalo de tempo entre a data do diagnóstico e a data do primeiro tratamento, sendo considerado como atraso para o início do tratamento o período acima de 60 dias, baseado na Lei Federal no 12.732 10. As variáveis sociodemográficas analisadas foram: sexo, faixa etária, raça/cor da pele, escolaridade, estado conjugal (com companheiro, isto é, casado ou com união consensual, e sem companheiro), concordância entre município de atendimento e município de residência, período temporal (triênio). As variáveis clínicas investigadas foram: local do tumor no cólon, morfologia, modalidade do primeiro tratamento e estadiamento de grupo (I, II, III e IV). Também foi utilizada a localização dos casos pela unidade federativa e macrorregiões brasileiras de acordo com a análise realizada.

A qualidade dos dados foi avaliada segundo o percentual de incompletude das variáveis estudadas, de acordo com o escore elaborado por Mello Jorge et al. 13. Segundo esse critério, completude excelente é considerada quando há até $10 \%$ de dados sem preenchimento; boa completude é classificada quando os percentuais variam entre $10 \%$ a $29,9 \%$; e má completude é considerada quando a falta de preenchimento supera $30 \%$. Sendo assim, informações quanto à escolaridade dos pacientes na Região Centro-oeste e ao estadiamento nas regiões Nordeste, Norte e Centro-oeste apresentaram alta proporção de incompletude, logo, optou-se por não as incluir no modelo logístico. 


\section{Análise estatística}

A análise descritiva foi composta de medidas como as seguintes: média, mediana e valores de mínimo e máximo. Para variáveis categóricas, foram calculadas frequência absoluta e relativa. $\mathrm{O}$ tempo mediano de início de tratamento também foi expresso segundo instituições hospitalares por unidades federativas do país. Tal unidade de análise deve-se pelo fato de buscar observar a distribuição espacial do tempo para o tratamento de acordo com a localização do hospital.

Para analisar a associação entre as variáveis independentes e o desfecho, optou-se por utilizar uma classe dos modelos lineares generalizados da família binomial, a regressão logística, considerando como desfecho uma resposta binária com as seguintes categorias: (a) tempo para início do tratamento igual ou superior a 60 dias; (b) tempo inferior a 60 dias.

Foi realizada a regressão logística simples a fim de avaliar a associação bruta de cada variável com o desfecho. Posteriormente, foi realizada a regressão logística múltipla. A escolha do modelo mais parcimonioso ocorreu por meio do método de seleção backward, com base no critério de informação de Akaike (AIC) e relevância teórica de cada variável. Além disso, foi considerada a completude das variáveis de acordo com Mello Jorge et al. 13; assim, variáveis com incompletude até 30\% foram analisadas. As medidas de associação calculadas foram odds ratio (OR) com intervalo de $95 \%$ de confiança (IC95\%). Para observar se havia outliers que poderiam impactar a qualidade do modelo proposto, foi realizada a análise de resíduos por meio da distância de Cook. Todas as análises estatísticas foram realizadas com o software $\mathrm{R}$ (http://www.r-project.org).

\section{Resultados}

\section{Análise descritiva da população de estudo}

O estudo incluiu 79.147 casos analíticos, com 39.133 (49,44\%) de câncer de cólon e 40.014 (50,56\%) de câncer retal (Figura 1). Os casos registrados foram oriundos de 263 unidades hospitalares, sendo 39,5\% localizadas na Região Sudeste, 25,5\% na Região Sul, 21,7\% na Região Nordeste, 8,4\% na Região Centro-oeste e 4,9\% na Região Norte.

Quanto a variáveis sociodemográficas dos casos de câncer no cólon, destaca-se que 28,3\% tinham idade entre 60 a 69 anos, 61,5\% eram brancos ou pardos (32,5\%). Uma parcela elevada dos casos tinha baixa escolaridade, sendo 7,8\% analfabetos e 64,6\% com Ensino Fundamental (completo ou incompleto). Quanto à situação conjugal, predominou a categoria denominada "com companheiro" (60,2\%). A Região Sudeste foi responsável pelo maior percentual de casos registrados (57,5\%), ao passo que as regiões Norte e Centro-oeste apresentaram 2\% e 3,1\% dos casos, respectivamente. O tratamento ocorreu em um município diferente da residência do paciente em 51,9\% dos casos. A grande maioria dos pacientes $(79,2 \%)$ foi oriunda do SUS. Em 54\% dos pacientes, o tratamento ocorreu na mesma instituição do diagnóstico (Tabela 1).

De forma semelhante ao câncer de cólon, notou-se maior proporção de casos de câncer de reto com idade entre 60 e 69 anos (27,7\%). Grandes proporções ocorreram entre brancos $(58,5 \%)$ e pardos (35,1\%). Quanto à escolaridade, houve predominância de casos com baixa escolaridade (65,8\%) e analfabetos (10,5\%). Além dessas características, os pacientes com câncer de reto tinham em sua maioria companheiro (60,4\%), eram residentes da Região Sudeste (53,4\%), foram tratados em um município diferente de sua residência (58,2\%) e encaminhados do SUS (80,2\%). Em contraste com o observado para câncer do cólon, notou-se que $65,7 \%$ dos pacientes com câncer de reto foram encaminhados ao hospital com diagnóstico prévio (Tabela 1).

O primeiro procedimento terapêutico foi cirurgia na maioria dos casos, porém a radioterapia $(23,4 \%)$ e a quimioterapia $(27,8 \%)$ foram mais frequentes no câncer de reto. Houve o predomínio do estadiamento avançado (III e IV), sendo 60,8\% em casos de câncer de cólon e 57,7\% em casos de câncer de reto (Tabela 2). 
Tabela 1

Características sociodemográficas de pacientes com câncer de cólon e reto. Brasil, 2006-2015.

\begin{tabular}{|c|c|c|}
\hline Variáveis sociodemográficas & $\begin{array}{l}\text { Câncer de cólon } \\
\qquad \begin{array}{c}(\mathrm{n}=39.133) \\
\mathrm{n}(\%)\end{array}\end{array}$ & $\begin{array}{c}\text { Câncer de reto } \\
\qquad \begin{array}{c}(n=40.014) \\
n(\%)\end{array}\end{array}$ \\
\hline \multicolumn{3}{|l|}{ Sexo } \\
\hline Masculino & $18.426(47,1)$ & $21.138(52,8)$ \\
\hline Feminino & $20.703(52,9)$ & $18.874(47,2)$ \\
\hline \multicolumn{3}{|l|}{ Faixa etária (anos) } \\
\hline$<40$ & $2.490(6,6)$ & $2.519(6,4)$ \\
\hline $40-49$ & $4.788(12,7)$ & $4.878(12,5)$ \\
\hline $50-59$ & $8.907(23,6)$ & $9.455(24,2)$ \\
\hline $60-69$ & $10.687(28,3)$ & $10.803(27,7)$ \\
\hline $70-79$ & $8.534(22,6)$ & $8.685(22,2)$ \\
\hline$\geq 80$ & $2.294(6,1)$ & $2.725(7,0)$ \\
\hline \multicolumn{3}{|l|}{ Raça/Cor da pele } \\
\hline Branca & $14.047(61,5)$ & $15.177(58,5)$ \\
\hline Preta & $1.094(4,8)$ & $1.280(4,9)$ \\
\hline Amarela & $264(1,2)$ & $348(1,3)$ \\
\hline Parda & $7.413(32,5)$ & $9.109(35,1)$ \\
\hline Indígena & $23(0,1)$ & $33(0,1)$ \\
\hline \multicolumn{3}{|l|}{ Escolaridade } \\
\hline Analfabeto & $2.245(7,8)$ & $3.133(10,5)$ \\
\hline Ensino Fundamental incompleto & $12.441(43,3)$ & $13.498(45,2)$ \\
\hline Ensino Fundamental completo & $6.105(21,3)$ & $6.153(20,6)$ \\
\hline Ensino Médio & $5.168(18,0)$ & $4.861(16,3)$ \\
\hline Ensino Superior & $2.747(9,6)$ & $2.243(7,5)$ \\
\hline \multicolumn{3}{|l|}{ Estado conjugal } \\
\hline Com companheiro & $12.600(60,2)$ & $14.898(60,4)$ \\
\hline Sem companheiro & $8.323(39,8)$ & $9.767(39,6)$ \\
\hline \multicolumn{3}{|l|}{ Regiões } \\
\hline Centro-oeste & $1.208(3,1)$ & $1.275(3,2)$ \\
\hline Nordeste & $5.069(13,0)$ & $6.835(17,1)$ \\
\hline Norte & $796(2,0)$ & $1.134(2,8)$ \\
\hline Sudeste & $22.501(57,5)$ & $21.378(53,4)$ \\
\hline Sul & $9.559(24,4)$ & $9.392(23,5)$ \\
\hline \multicolumn{3}{|l|}{ Período do diagnóstico } \\
\hline $2006-2008$ & $8.753(22,4)$ & $9.462(23,6)$ \\
\hline $2009-2011$ & $12.556(32,1)$ & $13.144(32,8)$ \\
\hline $2012-2015$ & $17.824(45,5)$ & $17.408(43,5)$ \\
\hline \multicolumn{3}{|l|}{ Município de atendimento } \\
\hline Mesmo de residência & $18.807(48,1)$ & $16.734(41,8)$ \\
\hline Município diferente & $20.326(51,9)$ & $23.280(58,2)$ \\
\hline \multicolumn{3}{|l|}{ Diagnóstico anterior } \\
\hline Sem diagnóstico & $21.151(54,0)$ & $13.713(34,3)$ \\
\hline Com diagnóstico & $17.982(46,0)$ & $26.301(65,7)$ \\
\hline \multicolumn{3}{|l|}{ Origem do encaminhamento } \\
\hline SUS & $22.699(79,2)$ & $24.545(80,2)$ \\
\hline Convênio ou particular & $5.953(20,8)$ & $6.049(19,8)$ \\
\hline
\end{tabular}

SUS: Sistema Único de Saúde. 
Tabela 2

Características clínicas de pacientes com câncer de cólon e reto. Brasil, 2006-2015.

\begin{tabular}{lcc}
\hline Variáveis clínicas & $\begin{array}{c}\text { Câncer de cólon } \\
\mathbf{( n = 3 9 . 1 3 3 )} \\
\mathbf{n}(\%)\end{array}$ & $\begin{array}{c}\text { Câncer de reto } \\
\mathbf{( n = 4 0 . 0 1 4 )}\end{array}$ \\
& $\mathbf{n}(\%)$ \\
\hline Local do tumor no cólon & $5.648(24,8)$ & - \\
Cólon direito & $2.701(11,9)$ & - \\
Cólon transverso & $2.836(12,4)$ & - \\
Cólon esquerdo & $11.595(50,9)$ & - \\
Não especificado & & $29.004(72,5)$ \\
Morfologia & $25.970(66,4)$ & $6.062(15,1)$ \\
Adenocarcinoma, SOE & $6.894(17,6)$ & $851(2,1)$ \\
Adenocarcinoma tubular & $1.708(4,4)$ & $4.097(10,2)$ \\
Adenocarcinoma mucinoso & $4.561(11,7)$ & $18.773(48,7)$ \\
Outros & & $9.034(23,4)$ \\
Primeiro procedimento terapêutico & $28.678(77,6)$ & $10.731(27,8)$ \\
Cirurgia & $530(1,4)$ & \\
Radioterapia & $7.764(21,0)$ & $3.412(12,4)$ \\
Quimioterapia & & $8.264(30,0)$ \\
Estadiamento & $2.786(9,9)$ & $9.526(34,6)$ \\
I & $8.208(29,3)$ & $6.367(23,1)$ \\
II & $8.964(32,0)$ & \\
III & $8.080(28,8)$ & \\
IV & & \\
\hline
\end{tabular}

SOE: sem outra especificação.

\section{Tempo para o início do tratamento}

O tempo entre a data do diagnóstico e o início do tratamento no Brasil foi de 44 dias em média para câncer de cólon, com mediana de 21 dias. Para o câncer de reto, foi de 41 dias em média, com mediana em 60 dias. Aproximadamente $22 \%$ dos pacientes com câncer de cólon e 35\% dos casos com câncer de reto tiveram atraso para o tratamento acima de 60 dias. Enquanto as regiões Nordeste e Norte apresentaram maior tempo de espera para ambos os tipos de câncer, o menor intervalo de tempo entre o diagnóstico e o tratamento ocorreu na região Sudeste para o câncer de cólon. Em todas as regiões do Brasil, o tempo mediano para o início do tratamento do câncer de reto foi superior ao do câncer de cólon.

Para o câncer de cólon, os estados do Pará e do Rio de Janeiro apresentaram tempo de espera para o início do tratamento acima de 60 dias, com medianas de 70 dias e 64 dias, respectivamente (Figura 2a). Para o câncer de reto, as Unidades Federativas com elevado tempo de espera (mediana) foram: Rio de Janeiro (83 dias), Sergipe (74 dias), Pará (71 dias), Amazonas (66 dias) e Distrito Federal (62,5 dias) (Figura 2b).

Em relação ao tratamento realizado, observou-se que os pacientes com câncer de cólon e reto, cujo primeiro tratamento foi cirurgia, obtiveram tempo de espera reduzido em comparação àqueles que necessitaram de radioterapia e quimioterapia. Esse período foi menor para pacientes com câncer de cólon. Quanto às regiões, o maior tempo mediano de espera ocorreu na região Norte para radioterapia (70 dias) e quimioterapia (61 dias). Além disso, notou-se que o tempo para início do tratamento de quimioterapia e radioterapia foi semelhante para ambos os tipos de câncer (Figura 3). 


\section{Figura 2}

Distribuição espacial do tempo mediano para início do tratamento de câncer de cólon e reto segundo Unidade da Federação. Brasil, $2006-2015$.

2a) Câncer de cólon

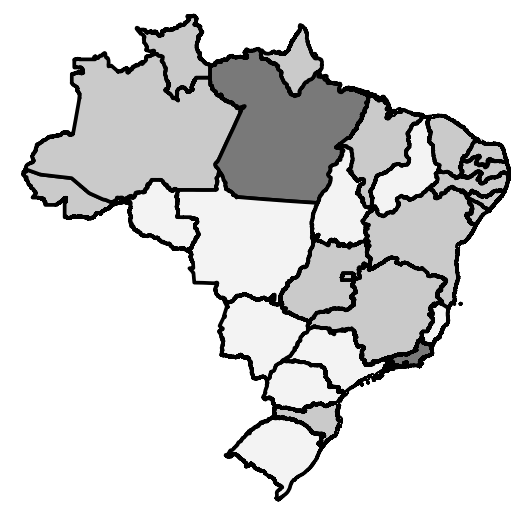

2b) Câncer de reto

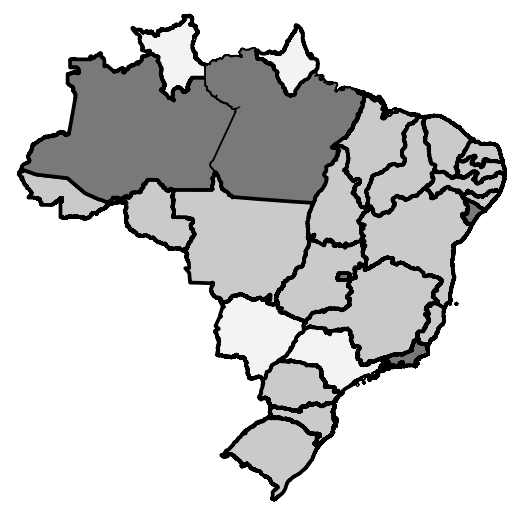

Tempo mediano (dias)

$\square$ Até 30

$\square^{30-60}$

Acima de 30

\section{Figura 3}

Tempo para início do tratamento de câncer de cólon e reto segundo modalidade terapêutica. Brasil, 2006-2015.

3a) Câncer de cólon

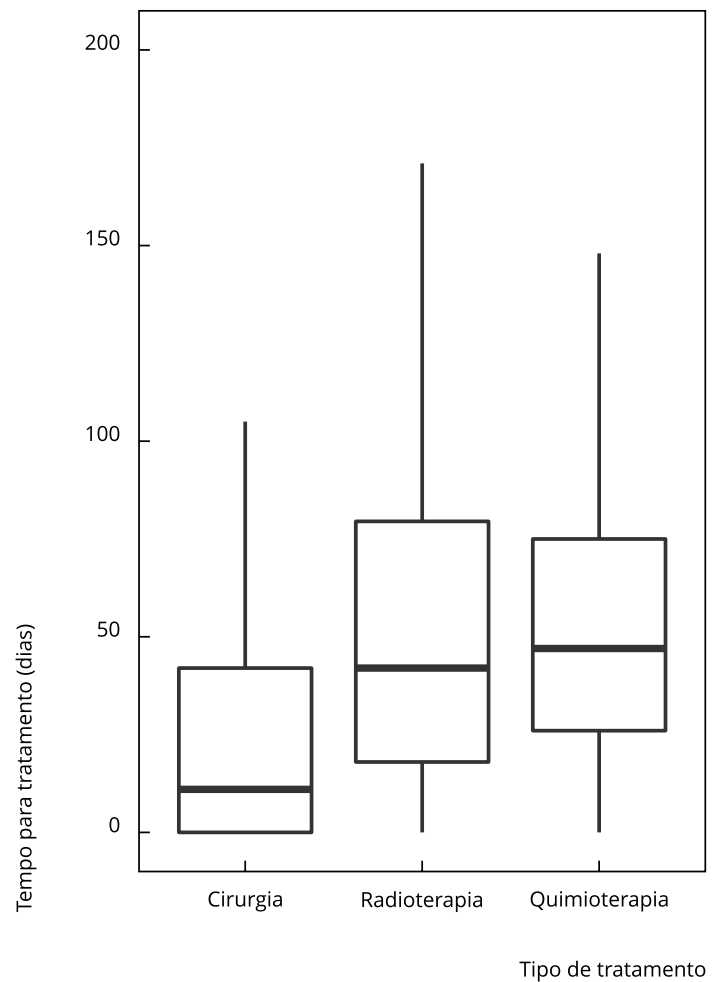

3b) Câncer de reto

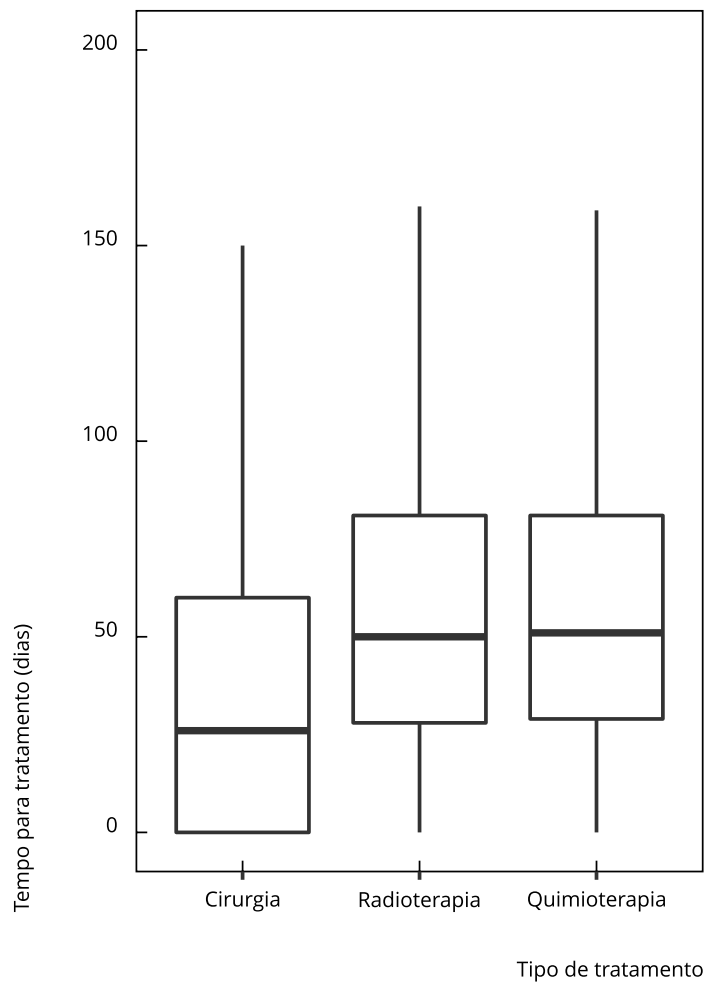


Após a seleção de melhor modelo, apenas as variáveis sexo e ano do diagnóstico não permaneceram no modelo estatístico com nível de 5\% de significância. Em relação aos fatores sociodemográficos dos pacientes com câncer de cólon, observou-se maior chance de atraso para o tratamento em indivíduos com idade acima de 60 anos, pacientes de raça/cor de pele preta (OR = 1,50; IC95\%: 1,21-1,84) e parda (OR = 1,28; IC95\%: 1,17-1,42), analfabetos (OR = 1,50; IC95\%: 1,19-1,90) e casos com baixa escolaridade. Notou-se também que, conforme o aumento da escolaridade, menor é a chance para o atraso do tratamento. Além disso, o tempo de espera foi maior do que o estipulado para pacientes sem companheiro (OR = 1,20; IC95\%: 1,09-1,31), e cujo tratamento ocorreu em um município distinto de sua residência (OR = 1,25; IC95\%:1,14-1,38). Em relação ao estadiamento, foi encontrada uma menor chance de atraso para o tratamento em casos com estádio avançado IV (OR = 0,82; IC95\%:0,68-1,00) quando comparado ao estadiamento I. Esses resultados estão sumarizados na Tabela 3.

Comparando as regiões do Brasil, a variável sexo se mostrou associada com o desfecho apenas para a Região Nordeste, sendo o sexo feminino um fator de proteção (OR = 0,84; IC95\%: 0,72-0,98). Residentes da Região Sudeste com idade acima de 60 anos apresentaram maior chance de atraso no tratamento. A raça/cor da pele preta apresentou significância estatística para a Região Sudeste $(O R=$ 1,45; IC95\%: 1,04-2,00). Comparados a pacientes com Ensino Superior, pacientes analfabetos e com baixa escolaridade tiveram maior chance de ter atraso para o tratamento nas regiões Sudeste, Sul e Nordeste. O estado conjugal "sem companheiro" mostrou força de associação semelhante nas regiões Sudeste (OR = 1,20; IC95\%: 1,03-1,40), Sul (OR = 1,19; IC95\%: 1,04-1,36) e Nordeste (OR = 1,21; IC95\%: 1,04-1,40). Em relação aos casos registrados de 2006 a 2008, os pacientes das regiões Sudeste e Sul diagnosticados durante os anos 2012 a 2015 tiveram maior chance de ter atraso no tratamento, ao passo que, na Região Norte, foi observada menor chance (OR = 0,49; IC95\%: 0,33-0,75). Em comparação aos pacientes com estadiamento I, os demais casos apresentaram menor OR para o atraso no tratamento na Região Sudeste (Tabelas 4 e 5).

\section{Fatores associados ao tempo de tratamento para câncer de reto}

Os fatores associados ao atraso do tratamento de câncer de reto no Brasil foram: idade acima de 60 anos, raça/cor da pele preta (OR = 1,44; IC95\%: 1,20-1,72) e parda (OR = 1,29; IC95\%: 1,19-1,39), analfabetismo (OR = 1,71; IC95\%: 1,40-2.09) e baixa escolaridade. Além desses fatores, notou-se também maior chance de atraso para o tratamento em indivíduos sem companheiro (OR = 1,13; IC95\%: 1,05-1,22), com diagnóstico entre 2009-2011 (OR = 1,19; IC95\%: 1,06-1,32) e 2012-2015 (OR = 1,19; IC95\%: 1,08-1,32), cujo tratamento foi realizado em um município distinto de sua residência $(\mathrm{OR}=$ 1,35; IC95\%: 1,25-1,47) e com menor chance de atraso em pacientes com estadiamento avançado IV $(\mathrm{OR}=0,78$; IC95\%: 0,68-0,91) (Tabela 3).

Em relação às regiões do Brasil, apenas para a Região Sudeste a variável sexo se manteve significativa, com maior chance para atraso do tratamento em mulheres (OR = 1,17; IC95\%: 1,03-1,33). Indivíduos com idade acima de 60 anos apresentaram maior chance de atraso para o tratamento em todas as regiões brasileiras, exceto no Nordeste. Obtiveram mais chance de iniciar o tratamento acima de 60 dias pacientes de raça/cor da pele preta (OR = 1,62; IC95\%: 1,26-2,08) e parda (OR = 1,20; IC95\%: 1,05-1,38) na Região Sudeste; casos com baixa escolaridade ou analfabetos nas regiões Sudeste, Sul e Nordeste; sem companheiros nas regiões Sul (OR = 1,21; IC95\%: 1,08-1,36) e Nordeste (OR = 1,13; IC95\%: 1,00-1,27), com ano de diagnóstico entre 2012 a 2015 na Região Sudeste e, a partir de 2009 no Nordeste. Pacientes que realizaram o tratamento em um município distinto de sua residência tiveram elevadas chances de iniciar o tratamento acima de 60 dias nas regiões Sudeste $(\mathrm{OR}=1,36$; IC95\%: 1,19-1,55), Nordeste (OR = 1,55; IC95\%: 1,37-1,76) e Centro-oeste (OR = 1,85; IC95\%: 1,442,38). Além disso, notou-se menor chance de atraso para casos com estadiamento II, II, e IV quando comparados ao estadiamento I, na Região Sudeste (Tabelas 6 e 7). 
Tabela 3

Fatores associados ao intervalo de tempo > 60 dias para o tratamento do câncer de cólon e reto. Brasil, 2006-2015.

\begin{tabular}{|c|c|c|c|c|}
\hline \multirow[t]{2}{*}{ Variáveis explicativas } & \multicolumn{2}{|c|}{ Câncer de cólon } & \multicolumn{2}{|c|}{ Câncer de reto } \\
\hline & $\begin{array}{l}\text { OR bruta } \\
\text { (IC95\%) }\end{array}$ & $\begin{array}{l}\text { OR ajustada * } \\
\text { (IC95\%) }\end{array}$ & $\begin{array}{l}\text { OR bruta } \\
\text { (IC95\%) }\end{array}$ & $\begin{array}{l}\text { OR ajustada * } \\
\text { (IC95\%) }\end{array}$ \\
\hline \multicolumn{5}{|l|}{ Sexo } \\
\hline Masculino & 1,00 & ** & 1,00 & ** \\
\hline Feminino & $1,01(0,96-1,06)$ & ** & $1,09(1,05-1,14)$ & ** \\
\hline \multicolumn{5}{|l|}{ Faixa etária (anos) } \\
\hline$<40$ & 1,00 & 1,00 & 1,00 & 1,00 \\
\hline $40-49$ & $1,21(1,07-1,37)$ & $1,09(0,86-1,37)$ & $1,21(1,08-1,34)$ & $1,15(0,95-1,39)$ \\
\hline $50-59$ & $1,30(1,16-1,46)$ & $1,32(1,07-1,64)$ & $1,36(1,23-1,50)$ & $1,36(1,14-1,62)$ \\
\hline $60-69$ & $1,42(1,27-1,58)$ & $1,38(1,12-1,70)$ & $1,45(1,32-1,60)$ & $1,34(1,13-1,60)$ \\
\hline $70-79$ & $1,49(1,33-1,67)$ & $1,47(1,18-1,83)$ & $1,56(1,41-1,72)$ & $1,43(1,20-1,72)$ \\
\hline$\geq 80$ & $1,78(1,55-2,04)$ & $1,29(1,00-1,66)$ & $2,21(1,97-2,48)$ & $1,55(1,26-1,91)$ \\
\hline \multicolumn{5}{|l|}{ Raça/Cor da pele } \\
\hline Branca & 1,00 & 1,00 & 1,00 & 1,00 \\
\hline Preta & $1,42(1,25-1,62)$ & $1,50(1,21-1,84)$ & $1,47(1,31-1,65)$ & $1,44(1,20-1,72)$ \\
\hline Amarela & $1,06(0,81-1,39)$ & $1,03(0,69-1,55)$ & $0,87(0,70-1,09)$ & $0,93(0,67-1,27)$ \\
\hline Parda & $1,20(1,13-1,28)$ & $1,28(1,17-1,42)$ & $1,20(1,14-1,26)$ & $1,29(1,19-1,39)$ \\
\hline \multicolumn{5}{|l|}{ Escolaridade } \\
\hline Analfabeto & $1,98(1,73-2,28)$ & $1,50(1,19-1,90)$ & $2,43(2,16-2,74)$ & $1,71(1,40-2,09)$ \\
\hline Ensino Fundamental incompleto & $1,82(1,63-2,03)$ & $1,45(1,20-1,76)$ & $1,90(1,72-2,11)$ & $1,53(1,29-1,82)$ \\
\hline Ensino Fundamental completo & $1,44(1,28-1,62)$ & $1,34(1,10-1,64)$ & $1,49(1,34-1,67)$ & $1,25(1,05-1,50)$ \\
\hline Ensino Médio & $1,42(1,26-1,61)$ & $1,17(0,95-1,44)$ & $1,43(1,28-1,60)$ & $1,21(1,00-1,46)$ \\
\hline Ensino Superior & 1,00 & 1,00 & 1,00 & 1,00 \\
\hline \multicolumn{5}{|l|}{ Estado conjugal } \\
\hline Com companheiro & 1,00 & 1,00 & 1,00 & 1,00 \\
\hline Sem companheiro & $1,14(1,08-1,22)$ & $1,20(1,09-1,31)$ & $1,16(1,10-1,22)$ & $1,13(1,05-1,22)$ \\
\hline \multicolumn{5}{|l|}{ Período do diagnóstico } \\
\hline $2006-2008$ & 1,00 & 1,00 & 1,00 & 1,00 \\
\hline 2009-2011 & $1,15(1,07-1,22)$ & $\star \star$ & $1,13(1,07-1,19)$ & $1,19(1,06-1,32)$ \\
\hline 2012-2015 & $1,22(1,15-1,30)$ & ** & $1,09(1,04-1,15)$ & $1,19(1,08-1,32)$ \\
\hline \multicolumn{5}{|l|}{ Município de atendimento } \\
\hline Mesmo de residência & 1,00 & 1,00 & 1,00 & 1,00 \\
\hline Município diferente & $1,39(1,33-1,46)$ & $1,25(1,14-1,38)$ & $1,30(1,24-1,35)$ & $1,35(1,25-1,47)$ \\
\hline \multicolumn{5}{|l|}{ Estadiamento } \\
\hline 1 & 1,00 & 1,00 & 1,00 & 1,00 \\
\hline II & $1,12(1,01-1,25)$ & $0,87(0,72-1,06)$ & $1,14(1,05-1,25)$ & $0,91(0,79-1,06)$ \\
\hline III & $1,15(1,03-1,28)$ & $0,85(0,70-1,02)$ & $1,20(1,10-1,30)$ & $0,97(0,84-1,11)$ \\
\hline IV & $1,11(0,99-1,24)$ & $0,82(0,68-1,00)$ & $0,98(0,89-1,07)$ & $0,78(0,67-0,91)$ \\
\hline
\end{tabular}

IC95\%: intervalo de 95\% de confiança; OR: odds ratio.

* Ajustada por: câncer de cólon - faixa etária, raça/cor da pele, escolaridade, estado conjugal, município de atendimento e estadiamento; câncer de reto

- faixa etária, raça/cor da pele, escolaridade, estado conjugal, período do diagnóstico, município de atendimento e estadiamento;

** Variável não permaneceu no modelo estatístico com nível de 5\% de significância. 
Tabela 4

Associação bruta entre os fatores socioeconômicos e o intervalo de tempo > 60 dias para o tratamento do câncer de cólon segundo tipo de tratamento. Brasil, 2006-2015.

\begin{tabular}{|c|c|c|c|c|c|}
\hline Variáveis explicativas & $\begin{array}{c}\text { Sudeste } \\
\text { OR bruta } \\
\text { (IC95\%) }\end{array}$ & $\begin{array}{c}\text { Sul } \\
\text { OR bruta } \\
\text { (IC95\%) }\end{array}$ & $\begin{array}{c}\text { Nordeste } \\
\text { OR bruta } \\
\text { (IC95\%) }\end{array}$ & $\begin{array}{c}\text { Norte } \\
\text { OR bruta } \\
\text { (IC95\%) }\end{array}$ & $\begin{array}{l}\text { Centro-oeste } \\
\text { OR bruta } \\
\text { (IC95\%) }\end{array}$ \\
\hline \multicolumn{6}{|l|}{ Sexo } \\
\hline Masculino & 1,00 & 1,00 & 1,00 & 1,00 & 1,00 \\
\hline Feminino & $1,03(0,96-1,09)$ & $0,95(0,87-1,05)$ & $0,94(0,84-1,06)$ & $1,14(0,85-1,54)$ & $1,17(0,89-1,55)$ \\
\hline \multicolumn{6}{|l|}{ Faixa etária (anos) } \\
\hline$<40$ & 1,00 & 1,00 & 1,00 & 1,00 & 1,00 \\
\hline $40-49$ & $1,49(1,23-1,80)$ & $1,28(0,98-1,67)$ & $0,99(0,77-1,27)$ & $1,21(0,68-2,13)$ & $1,18(0,66-2,12)$ \\
\hline $50-59$ & $1,63(1,36-1,95)$ & $1,45(1,13-1,87)$ & $1,17(0,93-1,48)$ & $1,58(0,96-2,62)$ & $0,82(0,47-1,43)$ \\
\hline $60-69$ & $1,88(1,58-2,25)$ & $1,42(1,11-1,82)$ & $1,18(0,94-1,48)$ & $1,58(0,96-2,60)$ & $1,13(0,65-1,94)$ \\
\hline $70-79$ & $1,95(1,64-2,33)$ & $1,60(1,24-2,05)$ & $1,23(0,98-1,56)$ & $1,22(0,71-2,10)$ & $1,08(0,62-1,89)$ \\
\hline$\geq 80$ & $3,58(2,87-4,45)$ & $1,33(1,01-1,75)$ & $1,13(0,84-1,50)$ & $1,33(0,62-2,89)$ & $0,74(0,37-1,51)$ \\
\hline \multicolumn{6}{|l|}{ Raça/Cor da pele } \\
\hline Branca & 1,00 & 1,00 & 1,00 & 1,00 & - \\
\hline Preta & $1,26(1,05-1,52)$ & $1,06(0,79-1,41)$ & $1,28(0,95-1,72)$ & $1,53(0,66-3,55)$ & $1,20(0,58-2,46)$ \\
\hline Amarela & $1,13(0,69-1,87)$ & $0,78(0,41-1,46)$ & $0,75(0,48-1,17)$ & $2,17(0,42-11,30)$ & $8,13(2,07-32,02)$ \\
\hline Parda & $0,89(0,80-0,98)$ & $0,99(0,78-1,25)$ & $1,20(1,02-1,41)$ & $0,89(0,57-1,38)$ & $0,84(0,62-1,15)$ \\
\hline \multicolumn{6}{|l|}{ Escolaridade } \\
\hline Analfabeto & $1,83(1,51-2,23)$ & $1,82(1,34-2,47)$ & $1,95(1,41-2,70)$ & $1,90(0,86-4,22)$ & * \\
\hline $\begin{array}{l}\text { Ensino Fundamental } \\
\text { incompleto }\end{array}$ & $1,80(1,56-2,08)$ & $1,95(1,54-2,47)$ & $1,69(1,26-2,27)$ & $1,50(0,75-2,98)$ & * \\
\hline Ensino Fundamental completo & $1,29(1,10-1,51)$ & $1,70(1,33-2,17)$ & $1,50(1,09-2,08)$ & $1,90(0,91-3,96)$ & * \\
\hline Ensino Médio & $1,41(1,20-1,65)$ & $1,43(1,10-1,87)$ & $1,23(0,89-1,69)$ & $1,98(0,94-4,16)$ & * \\
\hline Ensino Superior & 1,00 & 1,00 & 1,00 & 1,00 & * \\
\hline \multicolumn{6}{|l|}{ Estado conjugal } \\
\hline Com companheiro & 1,00 & 1,00 & 1,00 & 1,00 & 1,00 \\
\hline Sem companheiro & $1,10(1,00-1,20)$ & $1,11(0,98-1,25)$ & $1,17(1,03-1,33)$ & $0,92(0,67-1,27)$ & $1,15(0,83-1,59)$ \\
\hline \multicolumn{6}{|l|}{ Período do diagnóstico } \\
\hline $2006-2008$ & 1,00 & 1,00 & 1,00 & 1,00 & 1,00 \\
\hline $2009-2011$ & $1,21(1,11-1,33)$ & $0,98(0,86-1,13)$ & $1,10(0,92-1,31)$ & $0,85(0,54-1,34)$ & $0,84(0,59-1,18)$ \\
\hline $2012-2015$ & * & * & * & * & * \\
\hline \multicolumn{6}{|l|}{ Município de atendimento } \\
\hline Mesmo de residência & 1,00 & 1,00 & 1,00 & 1,00 & 1,00 \\
\hline Município diferente & $1,36(1,27-1,45)$ & $1,43(1,30-1,58)$ & $1,32(1,17-1,49)$ & $1,21(0,90-1,64)$ & $1,42(1,07-1,88)$ \\
\hline \multicolumn{6}{|l|}{ Estadiamento } \\
\hline 1 & 1,00 & 1,00 & * & * & * \\
\hline ॥ & $0,97(0,85-1,11)$ & $1,29(1,02-1,64)$ & * & * & * \\
\hline III & $1,00(0,87-1,14)$ & $1,32(1,04-1,67)$ & * & * & * \\
\hline IV & $1,00(0,87-1,15)$ & $1,15(0,91-1,46)$ & * & * & * \\
\hline
\end{tabular}

IC95\%: intervalo de 95\% de confiança; OR: odds ratio.

* Variável não foi inserida no modelo estatístico por falta de completude nos dados. 
Tabela 5

Fatores associados ao intervalo de tempo > 60 dias para o tratamento do câncer de cólon segundo regiões brasileiras. Brasil, 2006-2015.

\begin{tabular}{|c|c|c|c|c|c|}
\hline Variáveis explicativas & $\begin{array}{c}\text { Sudeste } \\
\text { OR ajustada * } \\
\text { (IC95\%) }\end{array}$ & $\begin{array}{c}\text { Sul } \\
\text { OR ajustada * } \\
\text { (IC95\%) }\end{array}$ & $\begin{array}{c}\text { Nordeste } \\
\text { OR ajustada * } \\
\text { (IC95\%) }\end{array}$ & $\begin{array}{c}\text { Norte } \\
\text { OR ajustada * } \\
\text { (IC95\%) }\end{array}$ & $\begin{array}{c}\text { Centro-oeste } \\
\text { OR ajustada * } \\
\text { (IC95\%) }\end{array}$ \\
\hline \multicolumn{6}{|l|}{ Sexo } \\
\hline Masculino & $* *$ & $\star \star$ & 1,00 & ** & ** \\
\hline Feminino & $* *$ & ** & $0,84(0,72-0,98)$ & ** & ** \\
\hline \multicolumn{6}{|l|}{ Faixa etária (anos) } \\
\hline$<40$ & 1,00 & $\star \star$ & ** & $* *$ & $* *$ \\
\hline $40-49$ & $1,69(1,07-2,67)$ & ** & ** & ** & ** \\
\hline $50-59$ & $2,03(1,32-3,11)$ & $\star *$ & ** & $* *$ & ** \\
\hline $60-69$ & $2,23(1,45-3,41)$ & $\star \star$ & ** & ** & ** \\
\hline $70-79$ & $2,69(1,71-4,10)$ & $\star \star$ & ** & ** & $* *$ \\
\hline$\geq 80$ & $2,74(1,70-4,44)$ & ** & ** & ** & ** \\
\hline \multicolumn{6}{|l|}{ Raça/Cor da pele } \\
\hline Branca & 1,00 & $\star \star$ & ** & $* *$ & 1,00 \\
\hline Preta & $1,45(1,04-2,00)$ & ** & ** & ** & $1,20(0,58-2,46)$ \\
\hline Amarela & $1,50(0,73-3,09)$ & $\star \star$ & ** & $* *$ & $8,13(2,07-32,02)$ \\
\hline Parda & $0,99(0,84-1,16)$ & $\star \star$ & ** & ** & $0,84(0,62-1,15)$ \\
\hline \multicolumn{6}{|l|}{ Escolaridade } \\
\hline Analfabeto & $1,74(1,18-2,57)$ & $1,52(1,06-2,18)$ & $1,73(1,24-2,43)$ & ** & $* * \star$ \\
\hline Ensino Fundamental incompleto & $1,63(1,21-2,19)$ & $1,77(1,35-2,32)$ & $1,49(1,10-2,02)$ & ** & 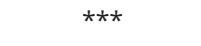 \\
\hline Ensino Fundamental completo & $1,43(1,03-1,98)$ & $1,85(1,39-2,46)$ & $1,43(1,03-2,01)$ & $* *$ & $* * *$ \\
\hline Ensino Médio & $1,12(0,81-1,56)$ & $1,40(1,03-1,90)$ & $1,09(0,79-1,52)$ & ** & $* * \star$ \\
\hline Ensino Superior & 1,00 & 1,00 & 1,00 & ** & $* * \star$ \\
\hline \multicolumn{6}{|l|}{ Estado conjugal } \\
\hline Com companheiro & 1,00 & 1,00 & 1,00 & ** & $* *$ \\
\hline Sem companheiro & $1,20(1,03-1,40)$ & $1,19(1,04-1,36)$ & $1,21(1,04-1,40)$ & ** & ** \\
\hline \multicolumn{6}{|l|}{ Município de atendimento } \\
\hline Mesmo de residência & 1,00 & 1,00 & 1,00 & $* *$ & 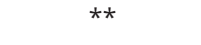 \\
\hline Município diferente & $1,22(1,04-1,42)$ & $1,36(1,19-1,55)$ & $1,30(1,11-1,51)$ & 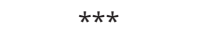 & $* * *$ \\
\hline \multicolumn{6}{|l|}{ Período do diagnóstico } \\
\hline $2006-2008$ & 1,00 & 1,00 & ** & 1,00 & $\star \star *$ \\
\hline 2009-2011 & $0,96(0,76-1,21)$ & $0,82(0,68-0,99)$ & $* * \star$ & $0,85(0,54-1,34)$ & $* * \star$ \\
\hline 2012-2015 & $1,28(1,04-1,58)$ & $1,10(0,94-1,30)$ & $* * *$ & $0,49(0,33-0,75)$ & $* * *$ \\
\hline \multicolumn{6}{|l|}{ Estadiamento } \\
\hline 1 & 1,00 & $\star \star$ & $* * *$ & $\star \star \star$ & $* * *$ \\
\hline II & $0,73(0,54-1,00)$ & $* * *$ & $* * *$ & $* * *$ & $* * *$ \\
\hline III & $0,63(0,47-0,86)$ & $* * *$ & $* * *$ & $\star \star \star *$ & $* * *$ \\
\hline IV & $0,67(0,49-0,91)$ & $\star \star \star *$ & $\star \star \star *$ & $\star \star \star *$ & 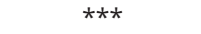 \\
\hline
\end{tabular}

IC95\%: intervalo de 95\% de confiança; OR: odds ratio.

* Ajustado por: Sudeste - faixa etária, raça/cor da pele, escolaridade, estado conjugal, período do diagnóstico, município de atendimento e estadiamento; Sul - escolaridade, estado conjugal, período do diagnóstico e município de atendimento; Nordeste - sexo, escolaridade, estado conjugal e período do diagnóstico; Norte - município de atendimento; Centro-oeste - raça/cor da pele;

** Variável não permaneceu no modelo estatístico com nível de 5\% de significância;

*** Variável não foi inserida no modelo estatístico por falta de completude nos dados. 
Tabela 6

Associação bruta entre os fatores socioeconômicos e o intervalo de tempo > 60 dias para o tratamento do câncer de reto segundo tipo de tratamento. Brasil, 2006-2015.

\begin{tabular}{|c|c|c|c|c|c|}
\hline Variáveis explicativas & $\begin{array}{c}\text { Sudeste } \\
\text { OR bruta } \\
\text { (IC95\%) }\end{array}$ & $\begin{array}{c}\text { Sul } \\
\text { OR bruta } \\
\text { (IC95\%) }\end{array}$ & $\begin{array}{c}\text { Nordeste } \\
\text { OR bruta } \\
\text { (IC95\%) }\end{array}$ & $\begin{array}{c}\text { Norte } \\
\text { OR bruta } \\
\text { (IC95\%) }\end{array}$ & $\begin{array}{l}\text { Centro-oeste } \\
\text { OR bruta } \\
\text { (IC95\%) }\end{array}$ \\
\hline \multicolumn{6}{|l|}{ Sexo } \\
\hline Masculino & 1,00 & 1,00 & 1,00 & 1,00 & 1,00 \\
\hline Feminino & $1,13(1,06-1,19)$ & $1,04(0,96-1,14)$ & $1,02(0,93-1,13)$ & $0,99(0,78-1,25)$ & $0,98(0,77-1,25)$ \\
\hline \multicolumn{6}{|l|}{ Faixa etária (anos) } \\
\hline$<40$ & 1,00 & 1,00 & 1,00 & 1,00 & 1,00 \\
\hline $40-49$ & $1,29(1,10-1,51)$ & $1,33(1,05-1,68)$ & $1,01(0,80-1,26)$ & $1,24(0,79-1,96)$ & $1,54(0,85-2,80)$ \\
\hline $50-59$ & $1,45(1,26-1,68)$ & $1,58(1,27-1,97)$ & $1,21(0,99-1,49)$ & $1,42(0,92-2,19)$ & $1,500,87-2,60)$ \\
\hline $60-69$ & $1,53(1,33-1,76)$ & $1,73(1,40-2,15)$ & $1,20(0,98-1,47)$ & $1,58(1,05-2,39)$ & $2,11(1,22-3,64)$ \\
\hline $70-79$ & $1,63(1,41-1,88)$ & $1,81(1,46-2,25)$ & $1,40(1,14-1,72)$ & $1,91(1,23-2,99)$ & $1,76(1,02-3,04)$ \\
\hline$\geq 80$ & $3,51(2,93-4,21)$ & $1,80(1,42-2,30)$ & $1,62(1,28-2,04)$ & $1,31(0,77-2,23)$ & $1,59(0,82-3,08)$ \\
\hline \multicolumn{6}{|l|}{ Raça/Cor da pele } \\
\hline Branca & 1,00 & 1,00 & 1,00 & 1,00 & - \\
\hline Preta & $1,40(1,19-1,64)$ & $0,98(0,74-1,29)$ & $1,23(0,95-1,60)$ & $0,75(0,34-1,66)$ & $2,19(1,09-4,41)$ \\
\hline Amarela & $0,72(0,45-1,15)$ & $0,94(0,56-1,56)$ & $0,90(0,65-1,24)$ & $0,48(0,05-4,68)$ & $0,99(0,38-2,59)$ \\
\hline Parda & $0,98(0,90-1,08)$ & $0,94(0,77-1,15)$ & $1,24(1,09-1,41)$ & $1,10(0,77-1,56)$ & $0,88(0,67-1,16)$ \\
\hline \multicolumn{6}{|l|}{ Escolaridade } \\
\hline Analfabeto & $2,30(1,95-2,72)$ & $1,83(1,41-2,39)$ & $3,09(2,28-4,17)$ & $1,58(0,79-3,18)$ & * \\
\hline $\begin{array}{l}\text { Ensino Fundamental } \\
\text { incompleto }\end{array}$ & $1,85(1,62-2,11)$ & $1,66(1,33-2,07)$ & $2,40(1,80-3,20)$ & $1,76(0,94-3,29)$ & * \\
\hline Ensino Fundamental completo & $1,46(1,26-1,68)$ & $1,44(1,14-1,81)$ & $1,70(1,25-2,32)$ & $1,57(0,80-3,08)$ & * \\
\hline Ensino Médio & $1,49(1,29-1,72)$ & $1,13(0,89-1,45)$ & $1,48(1,08-2,03)$ & $1,87(0,96-3,66)$ & * \\
\hline Ensino Superior & 1,00 & 1,00 & 1,00 & 1,00 & * \\
\hline \multicolumn{6}{|l|}{ Estado conjugal } \\
\hline Com companheiro & 1,00 & 1,00 & 1,00 & 1,00 & 1,00 \\
\hline Sem companheiro & $1,12(1,03-1,21)$ & $1,19(1,08-1,32)$ & $1,16(1,05-1,28)$ & $1,01(0,78-1,30)$ & $1,13(0,86-1,50)$ \\
\hline \multicolumn{6}{|l|}{ Período do diagnóstico } \\
\hline $2006-2008$ & 1,00 & 1,00 & 1,00 & 1,00 & 1,00 \\
\hline $2009-2011$ & $1,09(1,01-1,18)$ & $1,10(0,98-1,24)$ & $1,26(1,10-1,45)$ & $0,97(0,70-1,34)$ & $1,18(0,86-1,60)$ \\
\hline $2012-2015$ & $1,01(0,94-1,09)$ & $1,13(1,02-1,26)$ & $1,23(1,09-1,40)$ & $0,86(0,63-1,17)$ & $1,12(0,81-1,55)$ \\
\hline \multicolumn{6}{|l|}{ Município de atendimento } \\
\hline Mesmo de residência & 1,00 & 1,00 & 1,00 & 1,00 & 1,00 \\
\hline Município diferente & $1,16(1,10-1,23)$ & $1,30(1,19-1,42)$ & $1,69(1,52-1,86)$ & $1,04(0,83-1,32)$ & $1,86(1,45-2,39)$ \\
\hline \multicolumn{6}{|l|}{ Estadiamento } \\
\hline 1 & 1,00 & 1,00 & * & * & * \\
\hline ॥ & $1,06(0,95-1,18)$ & $1,22(1,01-1,48)$ & * & * & * \\
\hline III & $1,09(0,98-1,22)$ & $1,25(1,04-1,50)$ & * & * & * \\
\hline IV & $0,86(0,77-0,97)$ & $1,08(0,89-1,31)$ & * & * & * \\
\hline
\end{tabular}

IC95\%: intervalo de 95\% de confiança; OR: odds ratio.

* Variável não foi inserida no modelo estatístico por falta de completude nos dados. 
Tabela 7

Fatores associados ao intervalo de tempo > 60 dias para o tratamento do câncer de reto segundo tipo de tratamento, Brasil, 2006-2015) .

\begin{tabular}{|c|c|c|c|c|c|}
\hline Variáveis explicativas & $\begin{array}{c}\text { Sudeste } \\
\text { OR ajustada * } \\
\text { (IC95\%) }\end{array}$ & $\begin{array}{c}\text { Sul } \\
\text { OR ajustada * } \\
\text { (IC95\%) }\end{array}$ & $\begin{array}{c}\text { Nordeste } \\
\text { OR ajustada * } \\
\text { (IC95\%) }\end{array}$ & $\begin{array}{c}\text { Norte } \\
\text { OR ajustada * } \\
\text { (IC95\%) }\end{array}$ & $\begin{array}{c}\text { Centro-oeste } \\
\text { OR ajustada * } \\
\text { (IC95\%) }\end{array}$ \\
\hline \multicolumn{6}{|l|}{ Sexo } \\
\hline Masculino & 1,00 & ** & ** & ** & ** \\
\hline Feminino & $1,17(1,03-1,33)$ & ** & ** & ** & ** \\
\hline \multicolumn{6}{|l|}{ Faixa etária (anos) } \\
\hline$<40$ & 1,00 & 1,00 & ** & 1,00 & $* \star *$ \\
\hline $40-49$ & $1,22(0,87-1,69)$ & $1,37(1,00-1,87)$ & ** & $1,24(0,79-1,96)$ & $1,59(0,87-2,91)$ \\
\hline $50-59$ & $1,52(1,12-2,05)$ & $1,65(1,23-2,20)$ & ** & $1,42(0,92-2,19)$ & $1,55(0,89-2,69)$ \\
\hline $60-69$ & $1,57(1,16-2,13)$ & $1,76(1,32-2,35)$ & ** & $1,58(1,05-2,39)$ & $2,09(1,21-3,61)$ \\
\hline $70-79$ & $1,63(1,19-2,22)$ & $1,72(1,28-2,31)$ & ** & $1,91(1,23-2,99)$ & $1,80(1,04-3,13)$ \\
\hline$\geq 80$ & $1,98(1,39-2,81)$ & $1,60(1,15-2,22)$ & ** & $1,31(0,77-2,23)$ & $1,73(0,89-3,38)$ \\
\hline \multicolumn{6}{|l|}{ Raça/Cor da pele } \\
\hline Branca & 1,00 & $\star \star$ & ** & $\star \star$ & $* \star \star$ \\
\hline Preta & $1,62(1,26-2,08)$ & $* \star *$ & $\star \star \star *$ & $* * *$ & $* \star \star$ \\
\hline Amarela & $0,87(0,47-1,63)$ & $* * *$ & $\star \star *$ & $* \star *$ & $* \star *$ \\
\hline Parda & $1,20(1,05-1,38)$ & $* \star *$ & $\star \star \star$ & $* * *$ & $* * *$ \\
\hline \multicolumn{6}{|l|}{ Escolaridade } \\
\hline Analfabeto & $1,63(1,18-2,25)$ & $1,91(1,41-2,58)$ & $2,57(1,87-3,51)$ & $\star \star$ & $* \star \star$ \\
\hline Ensino Fundamental incompleto & $1,59(1,22-2,06)$ & $1,70(1,32-2,18)$ & $2,09(1,55-2,81)$ & $* \star \star$ & $\star \star *$ \\
\hline Ensino Fundamental completo & $1,34(1,01-1,77)$ & $1,58(1,22-2,05)$ & $1,54(1,11-2,12)$ & $\star * \star$ & $* \star *$ \\
\hline Ensino Médio & $1,36(1,02-1,82)$ & $1,24(0,94-1,64)$ & $1.38(1,00-1,91)$ & $* * *$ & $\star \star \star *$ \\
\hline Ensino Superior & 1,00 & 1,00 & 1,00 & $* * *$ & $* \star *$ \\
\hline \multicolumn{6}{|l|}{ Estado conjugal } \\
\hline Com companheiro & ** & 1,00 & 1,00 & $* \star *$ & $* \star *$ \\
\hline Sem companheiro & ** & $1,21(1,08-1,36)$ & $1,13(1,00-1,27)$ & $* * \star$ & $* \star \star$ \\
\hline \multicolumn{6}{|l|}{ Período do diagnóstico } \\
\hline $2006-2008$ & 1,00 & $\star \star$ & 1,00 & $\star \star$ & $\star \star \star *$ \\
\hline 2009-2011 & $1,16(0,97-1,40)$ & $\star \star \star$ & $1,33(1,12-1,57)$ & $* \star \star$ & $* \star \star$ \\
\hline $2012-2015$ & $1,35(1,14-1,61)$ & $* \star *$ & $1,26(1,08-1,48)$ & $* \star *$ & $* \star *$ \\
\hline \multicolumn{6}{|l|}{ Município de atendimento } \\
\hline Mesmo de residência & 1,00 & $* *$ & 1,00 & $\star \star$ & 1,00 \\
\hline Município diferente & $1,36(1,19-1,55)$ & $* \star *$ & $1,55(1,37-1,76)$ & $* * *$ & $1,85(1,44-2,38)$ \\
\hline \multicolumn{6}{|l|}{ Estadiamento } \\
\hline 1 & 1,00 & $\star \star$ & $\star \star$ & $* * *$ & $\star \star \star$ \\
\hline II & $0,67(0,52-0,85)$ & $\star \star \star$ & $\star \star \star *$ & $* * *$ & $* \star \star$ \\
\hline III & $0,72(0,57-0,91)$ & $* \star *$ & $* \star *$ & $* \star *$ & $* \star *$ \\
\hline IV & $0,50(0,39-0,64)$ & $\star \star \star$ & $\star \star *$ & $* * *$ & $* * *$ \\
\hline
\end{tabular}

IC95\%: intervalo de 95\% de confiança; OR: odds ratio.

* Ajustada por: Sudeste - sexo, faixa etária, raça/cor da pele, escolaridade, período do diagnóstico, município de atendimento e estadiamento; Sul faixa etária, escolaridade e estado conjugal; Nordeste - escolaridade, estado conjugal, período do diagnóstico e município de atendimento; Norte - faixa etária; Centro-oeste - faixa etária e município de atendimento;

** Variável não permaneceu no modelo estatístico com nível de 5\% de significância;

*** Variável não foi inserida no modelo estatístico por falta de completude nos dados. 


\section{Discussão}

Os achados do presente estudo evidenciaram o longo tempo de espera entre o diagnóstico e o tratamento de câncer de cólon e reto no Brasil. Também permitiram constatar a associação de fatores sociodemográficos e clínicos a um atraso para início do tratamento. Além disso, foi possível observar diferenças do tempo para o início do tratamento segundo macrorregiões do Brasil.

Pacientes de raça preta ou parda foram mais propensos ao atraso para o tratamento de câncer de cólon e reto. Possíveis causas para tal disparidade é o baixo nível socioeconômico 14,15 e o estadiamento avançado 15. Além disso, pacientes com câncer colorretal de raça preta enfrentam dificuldade no acesso ao rastreamento 16 , em consulta com especialista 17 , no recebimento do tratamento $17 \mathrm{e}$ experimentam maior tempo de espera para o início do tratamento 18 .

Analfabetos e pacientes com baixa escolaridade apresentaram maior tempo de espera para o tratamento. Isso se deve porque a baixa escolaridade pode interferir no acesso aos serviços de saúde, na compreensão das instruções médicas fornecidas durante confirmação diagnóstica e início do tratamento, na realização do autocuidado e no entendimento do tratamento prescrito 19,20.

Em ambos os tipos de câncer analisados, pessoas com idade acima de 60 anos tiveram mais chance de ter atraso no início do tratamento. As hipóteses para esse achado são: presença de comorbidades em pessoas com mais de 60 anos, presença de deficiências cognitivas, depressão, desnutrição, déficit funcional, negligência no autocuidado 21 e limitações na mobilidade 22 .

Houve maior chance de atraso ao tratamento de câncer de cólon e reto no Brasil em indivíduos sem companheiro, mas isso se evidencia para o câncer de cólon ao analisar as regiões Sudeste, Sul e Nordeste e para o câncer de reto nas regiões Sul e Nordeste. Pacientes com companheiros podem usufruir de maior apoio familiar e social. Consequentemente, esse fator impacta o bem-estar e a adaptação para o tratamento do câncer 23 .

Tendo em vista a criação da Lei no 12.732 em 22 de novembro de 2012, foi possível observar que não houve redução do tempo para o início do tratamento de câncer de cólon e reto no Brasil. Pacientes diagnosticados após 2012 apresentaram mais chance de atraso para o tratamento de câncer de cólon nas regiões Sudeste e Sul, e menos chance na Região Norte. Para o câncer de reto, houve mais chance de atraso no modelo referente ao Brasil e nas regiões Sudeste e Nordeste. Além da implementação da lei, é necessária a reestruturação da atenção oncológica. Caso contrário, o atraso no tratamento permanecerá decorrente dos seguintes fatores: atraso em agendamento de consultas médicas, exames patológicos e imuno-histoquímicos atrasados, falta de recursos humanos, escassez de quimioterápicos e aparelhos de radioterapia e ausência de hospitais 24 .

Quanto ao estadiamento do câncer, encontrou-se maior proporção de casos com estadiamento III e IV, totalizando 60,8\% dos casos de câncer de cólon e 57,6\% dos casos de câncer de reto. A alta proporção de pacientes com estadiamento avançado no momento do diagnóstico sugere falha no diagnóstico precoce, demonstrando, assim, deficiência na qualidade dos serviços de saúde no que se refere à sua oferta em tempo oportuno 25 .

A diferença entre municípios de residência do paciente e local do hospital se mostrou um importante fator para a explicação do tempo de espera acima de 60 dias para o tratamento de câncer de cólon e reto. Na literatura atual, o longo tempo de viagem até o hospital foi observado em indivíduos de baixa renda 26, negros e hispânicos 27 , com tratamento à base de radioterapia 28 . Em um sistema de saúde hierarquizado, pressupõe-se que haja o deslocamento do paciente para a busca de serviços de média e alta complexidade. No entanto, o tempo de deslocamento entre o município de residência e o município de atendimento foi avaliado em estudos anteriores, e concluiu-se que há concentração de unidades de alta complexidade e que os pacientes com câncer no Brasil enfrentam longas distâncias em busca de tratamento, principalmente nas regiões Norte e Nordeste 29,30.

O tempo mediano para início do tratamento do câncer de cólon e reto no Brasil foi abaixo de 60 dias, porém em algumas Unidades da Federação o tempo de espera foi superior a esse prazo, como na Região Norte (Pará e Amazonas), Nordeste (Sergipe e Bahia), Centro-oeste (Goiás e Distrito Federal) e Sudeste (Rio de Janeiro). Esse cenário pode ser devido à distribuição desigual dos serviços e/ou à sua falta de gestão. Em uma pesquisa sobre América Latina e Caribe, evidenciou-se que o Brasil concentra os serviços de oncologia nas principais cidades, nas quais se dispõe de infraestrutura e de médicos especializados para realizar a prevenção, o diagnóstico e o tratamento do câncer 31. Estudos interna- 
cionais encontraram associação entre o tempo de espera para o tratamento e regiões específicas. $\mathrm{O}$ tempo de espera é mais curto em regiões urbanas 32, com concentração de instituições privadas 33,34, e em regiões com populações sem privação econômica 35 .

Além dos fatores analisados, vale ressaltar a relevância de aspectos referentes à rede de atenção oncológica. Em um estudo de revisão, concluiu-se que a ausência de hospitais especializados, problemas no encaminhamento do paciente e dificuldades nos agendamentos de testes de diagnóstico afetam o tempo para o início do tratamento de câncer de mama 36 . A diferença entre hospitais gerais e hospitais especializados também é observada na taxa de mortalidade. Os hospitais especializados apresentam menor taxa de mortalidade hospitalar, e isso está associado ao maior volume de procedimentos e tecnologia disponíveis 37 .

Desde 2001, a Organização para a Cooperação e Desenvolvimento Econômico (OCDE) iniciou um projeto composto de 13 países a fim de diminuir o tempo de espera para o tratamento. Esses países definiram um tempo limite para o início do tratamento, e alguns deles implementaram estratégias que englobam toda a trajetória terapêutica do paciente. Mais particularmente, uma estratégia seria diminuir o tempo entre a consulta com médico geral e a consulta com um especialista, como, por exemplo, no Reino Unido, onde se estabeleceu o prazo de até duas semanas e aumentou-se a capacidade de prestação de serviços 38 . Além disso, todos os países mencionados têm um programa de rastreamento para o câncer de cólon e reto ${ }^{39}$, sendo possível detectar a doença antes da presença de sinais e sintomas e, possivelmente, em fase inicial. No contexto brasileiro, ações que visem a diminuir o tempo entre a consulta com o oncologista e a confirmação diagnóstica são importantes, uma vez que o acesso a exames diagnósticos é uma barreira no país.

Os repositórios de dados sobre câncer no Brasil tiveram atualizações no decorrer dos anos. Em relação ao registro de câncer de base hospitalar, marcos na legislação brasileira contribuíram para isso. A Portaria no 3.535 de 1998 estabeleceu que os Centros de Alta Complexidade em Oncologia devem manter o registro hospitalar de câncer. A Política Nacional de Atenção Oncológica, instituída por meio da Portaria no 2.439 em 2005, definiu parâmetros para a implantação e o funcionamento dos registros hospitalares de câncer. A Portaria no 741 de 2005 determinou que os dados anuais dos registros devem ser enviados para o INCA em setembro, a partir de 2007, que é responsável pela sistematização dos dados e divulgação 11. Em 2012, foi estabelecida a notificação compulsória dos casos de câncer pela Lei no 12.732. Além dos registros de câncer de base hospitalar, em 2019, foi construída a base de dados Painel-Oncologia, com o objetivo de fornecer uma ferramenta de rápido acesso a gestores, a fim de identificar atrasos entre o diagnóstico e o tratamento de câncer 40.

O estudo realizado englobou um grande número de participantes, totalizando 79.147 casos distribuídos em todas as macrorregiões brasileiras. A análise dos fatores associados ao atraso no tratamento foi realizada segundo a localização do tumor, considerando as diferenças anatômicas que interferem na modalidade terapêutica do câncer de cólon e reto 41. A definição de atraso para o início do tratamento baseou-se na Lei no 12.732, presente em todo o território brasileiro, possibilitando a comparação com outros estudos e contribuindo com a avaliação de políticas de saúde no Brasil.

Os dados analisados devem ser interpretados com cautela, devido a limitações do estudo. A população foi composta majoritariamente de pacientes tratados em instituições públicas, podendo representar um viés de seleção e a impossibilidade de avaliar a diferença do tempo para instituições públicas e privadas. Houve um número reduzido de casos para as regiões Norte e Centro-oeste, sendo possível que haja sub-registro de casos de câncer nessas regiões. A data do diagnóstico e do tratamento de câncer são dados frequentemente coletados nos registros hospitalares de câncer, colaborando, assim, para a comparação de estudos nacionais e internacionais, porém o tempo hábil para o início do tratamento é distinto, de acordo com a regulamentação de cada país, dificultando, assim, a comparação. Além disso, vale ressaltar que há outros períodos que interferem no tempo total para o acesso ao tratamento, como o intervalo entre os primeiros sintomas até a consulta com médico geral e o intervalo do tempo até a consulta com médico especialista, dados que não constam nos registros de câncer analisado.

Em síntese, devem receber maior atenção para iniciar o tratamento estratos populacionais que se encontram em maior vulnerabilidade, a partir de quaisquer destes fatores: idade maior que 60 anos, raça/cor de pele preta e parda, baixa escolaridade. Determinadas condições também são importantes, como o tratamento realizado em município distinto de sua residência, estado conjugal sem compa- 
nheiro e período do diagnóstico. Analisar o tempo de espera para o início do tratamento de câncer pode refletir deficiências no acesso ao tratamento e permite ressaltar a importância de medidas de saúde pública equânimes, a fim de diminuir iniquidades em saúde. Estudos prospectivos serão importantes para avaliar o tempo entre primeiros sintomas, consulta e triagem, além de analisar o impacto na sobrevida.

\section{Colaboradores}

M. A. N. Lima participou do desenho do estudo, da aquisição e curadoria dos dados secundários, da análise dos resultados, elaborou o artigo e aprovou a versão final. D. A. M. Villela participou do desenho do estudo, da análise dos resultados, elaborou o artigo e aprovou a versão final.

\section{Informações adicionais}

ORCID: Mariana Araujo Neves Lima (0000-00022063-484X); Daniel Antunes Maciel Villela (00000001-8371-2959).

\section{Agradecimentos}

Os autores agradecem os comentários de Marianna de Camargo Cancela e de Cléber Nascimento do Carmo.

\section{Referências}

1. Ferlay J, Colombet M, Soerjomataram I, Mathers C, Parkin DM, Piñeros M, et al. Estimating the global cancer incidence and mortality in 2018: GLOBOCAN sources and methods. Int J Cancer 2019; 144:1941-53.

2. Instituto Nacional de Câncer José Alencar Gomes da Silva. Estimativa 2020: incidência de câncer no Brasil. Rio de Janeiro: Instituto Nacional de Câncer José Alencar Gomes da Silva; 2020.

3. Medeiros GC, Bergmann A, Aguiar SS, Thuler LCS. Análise dos determinantes que influenciam o tempo para o início do tratamento de mulheres com câncer de mama no Brasil. Cad Saúde Pública 2015; 31:1269-82.

4. Silva IF, Silva IF, Koifman RJ. Cervical cancer treatment delays and associated factors in a cohort of women from a developing country. J Glob Oncol 2019; 5:1-11.

5. Sacramento RS, Simião LJ, Viana KCG, Andrade MAC, Amorim MHC, Zandonade E. Associação de variáveis sociodemográficas e clínicas com os tempos para início do tratamento do câncer de próstata. Ciênc Saúde Colet 2019; 24:3265-74.

6. Centers of Disease Control and Prevention. What are the risk factors for colorectal cancer? https://www.cdc.gov/cancer/colorectal/ basic_info/risk_factors.htm (acessado em 02/ Dez/2017). 
7. Instituto Brasileiro de Geografia e Estatística. Pesquisa Nacional de Saúde, 2013: percepção do estado de saúde, estilos de vida e doenças crônicas. Brasil, grandes regiões e Unidades da Federação. Rio de Janeiro: Instituto Brasileiro de Geografia e Estatística; 2014.

8. Arnold M, Sierra MS, Laversanne M, Soerjomataram I, Jemal A, Bray F. Global patterns and trends in colorectal cancer incidence and mortality. Gut 2017; 66:683-91.

9. Instituto Nacional de Câncer José Alencar Gomes da Silva. Atlas de mortalidade por câncer. Rio de Janeiro: Instituto Nacional de Câncer José Alencar Gomes da Silva; 2020.

10. Ministério da Saúde. Portaria no 876 , de 16 de maio de 2011. Dispõe sobre a aplicação da Lei no 12.732 , de 22 de novembro de 2012, que versa a respeito do primeiro tratamento do paciente com neoplasia maligna comprovada no âmbito do Sistema Único de Saúde (SUS). Diário Oficial da União 2019; 17 mai.

11. Instituto Nacional de Câncer José Alencar Gomes da Silva. Registros hospitalares de câncer: planejamento e gestão. 2a Ed. Rio de Janeiro: Instituto Nacional de Câncer José Alencar Gomes da Silva; 2010.

12. Ministério da Saúde. Portaria no 741, de 19 de dezembro de 2005. Definem as Unidades de Assistência de Alta Complexidade em Oncologia, os Centros de Assistência de Alta Complexidade em Oncologia (CACON) e os Centros de Referência de Alta Complexidade em Oncologia e suas aptidões e qualidades e inclui outras providências. Diário Oficial da União 2005; $23 \mathrm{dez}$.

13. Mello Jorge MHP, Gotlieb SLD, Oliveira H. O Sistema de Informação sobre Nascidos Vivos: primeira avaliação dos dados brasileiros. Inf Epidemiol SUS 1996; 2:15-48.

14. White AL, Vernon SW, Franzini L, Du XL. Racial disparities in colorectal cancer survival: to what extent are racial disparities explained by differences in treatment, tumor or hospital characteristics? Cancer 2010; 116:4622-31.

15. Gomez SL, O’Malley CD, Stroup A, Shema SJ, Satariano WA. Longitudinal, population-based study of racial/ethnic differences in colorectal cancer survival: impact of neighborhood socioeconomic status, treatment and comorbidity. BMC Cancer 2007; 7:193.

16. Lansdorp-Vogelaar I, Kuntz KM, Knudsen AB, van Ballegooijen M, Zauber AG, Jemal A. Contribution of screening and survival differences to racial disparities in colorectal cancer rates. Cancer Epidemiol Biomarkers Prev 2012; 21:728-36.

17. Simpson DR, Martínez ME, Gupta S, Hattangadi-Gluth J, Mell LK, Heestand G, et al. Racial Disparity in consultation, treatment, and the impact on survival in metastatic colorectal cancer. J Natl Cancer Inst 2013; 105:1814-20.
18. Jones LA, Ferrans CE, Polite BN, Brewer KC, Maker AV, Pauls HA, et al. Examining racial disparities in colon cancer clinical delay in the colon cancer patterns of care in Chicago Study. Ann Epidemiol 2017; 27:731-8.

19. Zarcos-Pedrinaci I, Fernández-López A, Téllez T, Rivas-Ruiz F, Rueda A, Morales SuarezVarela MM, et al. Factors that influence treatment delay in patients with colorectal cancer. Oncotarget 2017; 8:36728-42.

20. Zillmer JGV, Lima LM, Feijó AM, Schwartz E, Hisse CDN, Viegas ADC, et al. Caracterização dos clientes em tratamento radioterápico em um serviço no sul do Brasil. Rev Enferm UFSM 2013; 3:315-25.

21. Santos CA, Ribeiro AQ, Rosa COB, Ribeiro RCL. Depressão, déficit cognitivo e fatores associados à desnutrição em idosos com câncer. Ciênc Saúde Colet 2015; 20:751-60.

22. Alves LC, Leite IC, Machado CJ. Conceituando e mensurando a incapacidade funcional da população idosa: uma revisão de literatura. Ciênc Saúde Colet 2008; 13:1199-207.

23. Usta YY. Importance of social support in cancer patients. Asian Pac J Cancer Prev 2012; 13:3569-72.

24. Paulino E, Melo AC, Nogueira-Rodrigues A, Thuler LCS. Gynecologic cancer in Brazil and the law of sixty days. J Gynecol Oncol 2018; 29:e44.

25. Millas SG, Alawadi ZM, Wray CJ, Silberfein EJ, Escamilla RJ, Karanjawala BE, et al. Treatment delays of colon cancer in a safety-net hospital system. J Surg Res 2015; 198:311-6.

26. Scoggins JF, Fedorenko CR, Donahue SMA, Buchwald D, Blough DK, Ramsey SD. Is distance to provider a barrier to care for medicaid patients with breast, colorectal, or lung cancer? J Rural Health 2012; 28:54-62.

27. Guidry JJ, Aday LA, Zhang D, Winn RJ. Transportation as a barrier to cancer treatment. Cancer Pract 1997; 5:361-6.

28. Lin CC, Bruinooge SS, Kirkwood MK, Hershman DL, Jemal A, Guadagnolo BA, et al. Association between geographic access to cancer care and receipt of radiation therapy for rectal cancer. Int J Radiat Oncol Biol Phys 2016; 94:719-28.

29. Oliveira EXG, Melo ECP, Pinheiro RS, Noronha $\mathrm{CP}$, Carvalho MS. Acesso à assistência oncológica: mapeamento dos fluxos origemdestino das internações e dos atendimentos ambulatoriais. O caso do câncer de mama. Cad Saúde Pública 2011; 27:317-26.

30. Saldanha RF, Xavier DR, Carnavalli KM, Lerner K, Barcellos C, Saldanha RF, et al. Estudo de análise de rede do fluxo de pacientes de câncer de mama no Brasil entre 2014 e 2016. Cad Saúde Pública 2019; 35:e00090918.

31. Goss PE, Lee BL, Badovinac-Crnjevic T, Strasser-Weippl K, Chavarri-Guerra Y, Louis JS, et al. Planning cancer control in Latin America and the Caribbean. Lancet Oncol 2013; 14:391-436. 
32. Robertson S, Adolfsson J, Stattin P, Sjövall A, Winnersjö R, Hanning M, et al. Waiting times for cancer patients in Sweden: a nationwide population-based study. Scand J Public Health 2017; 45:230-7.

33. Pourcel G, Ledesert B, Bousquet P-J, Ferrari C, Viguier J, Buzyn A. Waiting times for cancer care in four most frequent cancers in several French regions in 2011 and 2012. Bull Cancer 2013; 100:1237-50.

34. Seiber EE, Camacho F, Zeeshan MF, Kern TT, Fleming S. Disparities in colorectal cancer treatment delay within appalachia - the role of for-profit hospitals. J Rural Health 2015; 31:382-91.

35. Lejeune C, Sassi F, Ellis L, Godward S, Mak V, Day M, et al. Socio-economic disparities in access to treatment and their impact on colorectal cancer survival. Int J Epidemiol 2010; 39:710-7.

36. Freitas AGQ, Weller M. Patient delays and system delays in breast cancer treatment in developed and developing countries. Ciênc Saúde Colet 2015; 20:3177-89.
37. Ramos MCA, Cruz LP, Kishima VC, Pollara WM, Lira ACO, Couttolenc BF, et al. Avaliação de desempenho de hospitais que prestam atendimento pelo sistema público de saúde, Brasil. Rev Saúde Pública 2015; 49:43.

38. Organisation for Economic Co-operation and Development. Cancer care: assuring quality to improve survival. Paris: Organisation for Economic Co-operation and Development; 2013. (OECD Health Policy Studies).

39. Schreuders EH, Ruco A, Rabeneck L, Schoen RE, Sung JJY, Young GP, et al. Colorectal cancer screening: a global overview of existing programmes. Gut 2015; 64:1637-49.

40. Atty ATM, Jardim BC, Dias MBK, Migowski A, Tomazelli JG. PAINEL-Oncologia: uma ferramenta de gestão. Rev Bras Cancerol 2020; 66:e-04827.

41. Hong TS, Clark JW, Haigis KM. Cancers of the colon and rectum: identical or fraternal twins? Cancer Discov 2012; 2:117-21. 
Abstract

Colorectal cancer presents high incidence worldwide, but case-fatality is higher in developing countries. The study's objective was to analyze sociodemographic and clinical factors associated with delay in the initiation of treatment for colorectal cancer in hospitals in Brazil. This is a retrospective study of data from hospital cancer registries in Brazil from 2006 to 2015. The target variable is time to initiation of treatment for colorectal cancer and possible associations between sociodemographic variables and clinical factors. The analysis revealed disparities in time to treatment according to sociodemographic strata and geographic regions. Higher odds of treatment delay were associated with age over 50 years, black race/color $(O R=1.50 ; 95 \% C I: 1.21-1.84)$ and brown race/color $(O R=1.28$; 95\%CI: 1.17-1.42), illiteracy or low schooling $(O R=1.50 ; 95 \% C I$ : 1.19-1.90), and treatment in a city far from the patient's residence $(O R=1.25$; 95\%CI: 1.14-1.38). For rectal cancer, higher odds of treatment delay were associated with age over 50 years, black $(O R=1.44 ; 95 \% C I: 1.20-1.72)$ or brown race) color $(O R=1.29 ; 95 \% C I: 1.19-1.39)$, illiteracy or low schooling $(\mathrm{OR}=1.71 ; 95 \% \mathrm{CI}: 1.40-2.09)$, and treatment in a city far from the patient's residence $(O R=1.35 ; 95 \% C I: 1.25-1.47)$. In conclusion, greater attention should be given to reducing the time to initiation of treatment in underprivileged regions and in social strata identified with barriers to timely treatment access.

Colon Neoplasms; Rectal Neoplasms; Time-toTreatment; Hospitalar Records; Health Services Accessibility

\section{Resumen}

El cáncer de colon y recto presenta una alta incidencia mundialmente, pese a que la letalidad de la enfermedad es mayor en paises en desarrollo. El objetivo de este estudio fue analizar los factores sociodemográficos y clínicos, asociados al retraso para el inicio del tratamiento de cáncer de colon y recto en hospitales en Brasil. Se trata de un estudio retrospectivo con datos de registros hospitalarios de cáncer en Brasil de 2006 a 2015. El resultado analizado es el tiempo para el inicio del tratamiento de cáncer de colon y recto, así como las posibles asociaciones entre variables sociodemográficas $y$ las relacionadas con factores clínicos. Se observó disparidades en el tiempo para el inicio del tratamiento, según estratos sociodemográficas y regiones geográficas. Existe una mayor oportunidad de retraso para el inicio del tratamiento de cáncer de colon en pacientes con una edad por encima de 50 años, de raza/afrodescendiente $(O R=1,50$; IC95\%: 1,21-1,84) y mulata/mestiza $(O R=1,28$; IC 95\%: 1,17-1,42), analfabetos $(O R=1,50$; IC95\%: 1,19-1,90) o con baja escolaridad, y cuyo tratamiento se produjo en un municipio distinto al de su residencia $(O R=1,25$; IC95\%: 1,14-1,38). En pacientes con cáncer de recto existe una mayor oportunidad de atraso para el inicio del tratamiento entre los casos con una edad por encima de 50 años, de raza/afrodescendiente $(O R=1,44$; IC95\%: 1,20-1,72) y mulata/mestiza $(O R=1,29$; IC95\%: 1,19-1,39), analfabetos $(O R=1,71$; IC95\%: 1,40-2,09) o con baja escolaridad, y cuyo tratamiento se produjo en un municipio distinto al de su residencia (OR = 1,35; IC95\%: 1,25-1,47). Como conclusión, se debe prestar mayor atención a la reducción del tiempo para comenzar el tratamiento en las regiones desfavorecidas y en estratos identificados con barreras de acceso al tratamiento en el tiempo adecuado.

Neoplasias del Cólon; Neoplasias del Recto; Tiempo de Tratamiento; Registros Hospitalares; Accesibilidad a los Servicios de Salud
Recebido em 09/Dez/2019

Versão final reapresentada em 10/Set/2020

Aprovado em 05/Out/2020 